М. ЛЕВНН, В. АРРО, Ю. ГИРШОВИЧ

\title{
НАИЛУЧШИЕ КВАДРАТУРНЫЕ ФОРМУЛЫ ДЛЯ НЕКОТОРЫХ МНОЖЕСТВ ФУНКЦИЙ
}

Квадратурная формула с остатком $R_{n}(f)$ называется наилучшей на множестве $H$ функций $f(x)$, если ее узлы и веса выбраны так, что величина

$$
\sup _{f \in H}\left|R_{n}(f)\right|
$$

достигает наименьшего значения [ $\left.{ }^{1}\right]$. Ниже рассматриваются задачи построения некоторых наилучших квадратурных формул, в частности, формул внда

$$
\int_{0}^{1} f(x) d x=\sum_{k=1}^{n} A_{k} f\left(x_{k}\right)+R_{n}(f) .
$$

Используем следующие обозначения: $g(x, t)--$ функция Грина для задачи

$$
\left\{\begin{array}{l}
y^{(2 r)}=\varphi(x) \\
y(0)=y(1)=y^{(2 i-1)}(0)=y^{(2 i-1)}(1)=0 \quad(i=1, \ldots, r-1)
\end{array}\right.
$$

$W^{(2 r)} L_{2}-$ множество заданных на отрезке $[0,1]$ функций $f(x), y$ которых $(2 r-1)$-я производная абсолютно непрерывна, а производная порядка $2 r$ удовлетворяет условию

$$
\left(\int_{0}^{1}\left|f^{(2 r)}(x)\right|^{2} d x\right)^{1 / 2} \leqslant M
$$

где $M$ - заданное число;

$W_{g}^{(2 r)} L_{2}-$ множество функций $f(x)$, принадлежащих множеству $W^{(2 r)} L_{2}$ и удовлетворяющих условиям

$$
f(0)=f(1)=f^{(2 i-1)}(0)=f^{(2 i-1)}(1)=0 \quad(i=1, \ldots, r-1) .
$$

Найдем наилучшую на множестве $W_{g}^{(2 r)} L_{2}$ формулу

$$
\int_{0}^{1} f(x) d x=\alpha_{r} f^{(2 r-1)}(0)+\sum_{k=1}^{n} A_{k} f\left(x_{k}\right)+\beta_{r} f^{(2 r-1)}(1)+R_{n}(f) .
$$

Легко проверить $\left[{ }^{2}\right]$, что

$$
g(x, t)=\frac{(x-t)^{2 r-1}}{(2 r-1) !} E(x-t)-H(x, t),
$$


Наилучиие квадратурные формуль...

113

где

$$
H(x, t)=\sum_{i=1}^{r-1} \lambda_{i}(t) x^{2 i}+\lambda_{r}(t) x^{2 r-1}
$$

а функции

$$
\lambda_{1}(t), \ldots, \lambda_{r}(t)
$$

выбраны из условия

$$
\begin{gathered}
H(1, t)=\frac{(1-t)^{2 r-1}}{(2 r-1) !}, \quad H_{x^{2 /-1}}^{(2 j-1)}(1, t)=\frac{(1-t)^{2 r-2 j}}{(2 r-2 j) !} \quad(j=1, \ldots, r-1) ; \\
E(u)= \begin{cases}0, & u \leqslant 0 \\
1, & u>0 .\end{cases}
\end{gathered}
$$

Функция $f(x)$, принадлежащая множеству $W_{g}^{(2 r)} L_{2}, \quad$ допускает представление

$$
f(x)=\int_{0}^{1} f^{(2 r)}(t) g(x, t) d t .
$$

Подставляя это представление в (3) и учитывая (4) и (5), получаем

$$
R_{n}(f)=\int_{0}^{1} f^{(2 r)}(t) K(t) d t,
$$

где

$$
\begin{gathered}
K(t)=\int_{0}^{1} g(x, t) d x-\sum_{k=1}^{n} A_{k} g\left(x_{k}, t\right)+ \\
+(2 r-1) ! \alpha_{r} \lambda_{r}(t)-\beta_{r}\left[1-(2 r-1) ! \lambda_{r}(t)\right] .
\end{gathered}
$$

Применение к (6) неравенства Буняковского дает неравенство

$$
\left|R_{n}(f)\right| \leqslant M\left(\int_{0}^{1}|K(t)|^{2} d t\right)^{1 / 2} .
$$

Введем в рассмотрение функцию

$$
\varphi(x)=\int_{0}^{1} K(t) g(x, t) d t .
$$

Так как для функции

$$
f_{0}(x)=M \varphi(x) /\|K(t)\|_{L_{2}},
$$

принадлежащей множеству $W_{g}^{(2 r)} L_{2}$, неравенство (8) превращаетсл в равенство, то этим

$$
\sup _{f \in W_{\mathbb{z}}^{(2 r)} L_{2}}\left|R_{n}(f)\right|=M\left(\int_{0}^{1}|K(t)|^{2} d t\right)^{1 / 2} .
$$

Итак, для построения наилучшей формулы (3) ее узлы и веса нужно выбрать так, чтобы величина

$$
U=\int_{0}^{1}|K(t)|^{2} d t
$$

приняла наименьшее значение.

Для решения этой задачи рассмотрим подробнее функцию (9). Из свойств функции Грина сразу следует, что 


$$
\varphi^{(2 r)}(t)=K(t),
$$

$1^{\circ} . \varphi(0)=\varphi(1)=\varphi^{(2 i-1)}(0)=\varphi^{(2 i-1)}(1)=0 \quad(i=1, \ldots, r-1)$.

Так как оператор

$$
\left\{\begin{array}{l}
y^{(2 r)} \\
y(0)=y(1)=y^{(2 i-1)}(0)=y^{(2 i-1)}(1)=0 \quad(i=1, \ldots, r-1)
\end{array}\right.
$$

является самосопряженным, то $g(x, t)=g(t, x)$, и, следовательно, $g(x, t)$ как функция от $t$ удовлетворяет условиям $1^{\circ}$, поэтому

$$
g_{t^{2 t-1}}^{(2 i-1)}(x, 0)=g_{t^{2 t-1}}^{(2 i-1)}(x, 1)=0 \quad(i=1, \ldots, r-1) .
$$

В то же время из равенства $g(x, t)=g(t, x)$ по (4) имеем

$$
g(x, t)=\frac{(t-x)^{2 r-1}}{(2 r-1) !} E(t-x)-\sum_{i=1}^{r-1} \lambda_{i}(x) t^{2 i}-\lambda_{r}(x) t^{2 r-1} .
$$

Так как для всех $t>0$

$$
g(0, t)=g_{x^{2 t-1}}^{(2 i-1)}(0, t)=0 \quad(i=1, \ldots, r-1),
$$

то из (14) следует, что

$$
\begin{aligned}
& \lambda_{r}(0)=\frac{1}{(2 r-1) !} \\
& \lambda_{r}^{(2 i-1)}(0)=0 \quad(i=1, \ldots, r-1) .
\end{aligned}
$$

Аналогично получаем, что

$$
\lambda_{r}(1)=\lambda_{\tau}^{(2 i-1)}(1)=0 \quad(i=1, \ldots, r-1) .
$$

Учитывая (13), (16) и (17), по (7) и (12) имеем

$$
2^{\circ} . \varphi^{(2 r+2 i-1)}(0)=\varphi^{(2 r+2 i-1)}(1)=0 \quad(i=1, \ldots, r-1) .
$$

Из (7) и (12) следует, что функция $\varphi(t)$ представима в виде

$$
\varphi(t)=\frac{t^{4 r}}{(4 r) !}+P_{4 r-1}(t)+\sum_{k=1}^{n} \mu_{k}\left(x_{k}-t\right)^{4 r-1} E\left(x_{k}-t\right),
$$

где $P_{4 r-1}(t)$ - некоторый многочлен степени $4 r-1$. Но тогда

$3^{\circ}$. Функция $\varphi(t)$ имеет на отрезке $[0,1]$ непрерывную производную порядка $4 r-2$ и на каждом отрезке $\left[0, x_{1}\right),\left[x_{1}, x_{2}\right), \ldots,\left[x_{n}, 1\right]$ есть многочлен степени $4 r$ со старшим коэффициентом $1 /(4 r)$ !.

Из условий

$$
\begin{aligned}
& U_{A_{k}}^{\prime}=U_{x_{k}}^{\prime}=0 \quad(k=1, \ldots, n), \\
& U_{\alpha_{r}}^{\prime}=U_{\beta_{r}}^{\prime}=0
\end{aligned}
$$

минимума величины (11) непосредственно вытекает следующее свойство соответствующей наилучшей формуле (3) функции $\varphi(x)$ :

$$
\begin{aligned}
4^{\circ} . & \varphi\left(x_{k}\right)=\varphi^{\prime}\left(x_{k}\right)=0 \quad(k=1, \ldots, n), \\
& \varphi^{(2 r-1)}(0)=\varphi^{(2 r-1)}(1)=0 .
\end{aligned}
$$


Пусть теперь

$$
z_{k}=k H \quad(k=0,1, \ldots, n+1), H=\frac{1}{n+1},
$$

$Q(x)$ - функция, которая на каждом отрезке $\left[z_{i}, z_{i+1}\right](i=0,1, \ldots, n)$ совпадает с функцией

$$
\frac{H^{4 r}}{(4 r) !}\left\lceil R\left(\frac{x-z_{i}}{H}\right)-B_{4 r}\right],
$$

где $B_{m}(x)$ - многочлен Бернулли степени $m, B_{m}=B_{m}(0)$.

Непосредственной проверкой убеждаемся, что функция $Q(x)$ удовлетворяет условиям $1^{\circ}-4^{\circ}$, если положить $x_{i}=z_{i}(i=1, \ldots, n)$.

Так как по теореме И. Шёнберга и С. Карлина $\left[{ }^{3}\right]^{*}$ существуют единственный набор узлов $0 \leqslant x_{1}<\ldots<x_{n} \leqslant 1$ и соответствующая ему функция (18), для которой выполнены условия $1^{\circ}-4^{\circ}$, то отсюда имеем следующее: узлами наилучшей формулы (3) являются значения

$$
x_{k}=\frac{k}{n+1} \quad(k=1, \ldots, n),
$$

а функция $\varphi(x)$, соответствующая наилучшей формуле (3), совпадает с $Q(x)$. Но тогда по (12)

$$
K(t)=Q^{(2 r)}(t)
$$

Ввиду того, что $g_{t^{2 r-1}}^{(2 r-1)}(x, x+0)-g t^{2 r-1}(x, x-0)=1$, имеем

$$
A_{k}=K^{(2 r-1)}\left(x_{k}-0\right)-K^{(2 r-1)}\left(x_{k}+0\right) \quad(k=1, \ldots, n) .
$$

Отсюда и по (20) получаем веса наилучшей формулы (3)

$$
A_{k}=H=\frac{1}{n+1} \quad(k=1, \ldots, n) .
$$

По (7) с учетом (15), (17) и того, что $g(x, 0)=g(x, 1)=0$, имеем

$$
K(0)=\alpha_{r}, \quad K(1)=-\beta_{r} .
$$

Но тогда по (20) получаем значения весов

$$
\alpha_{r}=-\beta_{r}=\frac{B_{2 r}}{(n+1)^{2 r}(2 r) !} .
$$

Итак, узлы и веса наилучшей формулы (3) найдены. Найдем точную оценку ошибки этой формулы. По (11) и (20)

$$
\begin{gathered}
U=\sum_{i=0}^{n} \int_{z_{i}}^{z_{i+1}}\left[\frac{H^{2 r}}{(2 r) !}\right]^{2} B_{2 r}^{2}\left(\frac{x-z_{i}}{H}\right) d x= \\
=\frac{H^{4 r+1}}{[(2 r) !]^{2}} \sum_{i=0}^{n} \int_{0}^{1} B_{2 r}^{2}(u) d u=\frac{-B_{4 r}}{(n+1)^{4 r} \cdot(4 r) !},
\end{gathered}
$$

и поэтому по (10)

* Доказательство этой теоремы см. в [4]. 


$$
\sup _{f \in W_{g}^{(2 r)} L_{2}}\left|R_{n}(f)\right|=\frac{M}{(n+1)^{2 r}} \sqrt{\frac{-B_{4 r}}{(4 r) !}} .
$$

Таким образом, доказана следующая

Те орем а 1. Единственная наилучшая на множестве $W_{\mathrm{g}}^{(2 r)} L_{2}$ квадратурная формула (3) имеет узль (19), веса (21) и (22), оценку остатка (23).

Введем следующие множества функций:

$$
\begin{aligned}
& W_{01}^{*(2 r)} L_{2}=\left\{f(x): f \in W_{g}^{(2 r)} L_{2}, f^{(2 r-1)}(0)=f^{(2 r-1)}(1)=0\right\}, \\
& \bar{W}_{01}^{(2 r)} L_{2}=\left\{f(x): f \in W^{(2 r)} L_{2}, f^{(2 i-1)}(0)=f^{(2 i-1)}(1)=0 \quad(i=1, \ldots, r)\right\}, \\
& W_{01}^{(2 r)} L_{2}=\left\{f(x): f \in W^{(2 r)} L_{2}, f^{(2 i-1)}(0)=f^{(2 i-1)}(1) \quad(i=1, \ldots, r)\right\} .
\end{aligned}
$$

Из теоремы 1 и работы [5] получаем следующее утверждение.

Т е орем а 2. Единственная наилучшая на множестве $W_{01}^{*(2 r)} L_{2}$ квадратурная формула (1) имеет узль (19), веса (21), оценку остатка, равную значению (23).

Из теоремы 2 и [ $\left.{ }^{5}\right]$ следует

Т е орем а 3. Единственная наилучшая на множестве $\bar{W}_{01}^{(2 r)} L_{2}$ квадратурная формула

$$
\int_{0}^{1} f(x) d x=B f(0)+\sum_{k=1}^{n} A_{k} f\left(x_{k}\right)+C f(1)+R_{n}(f)
$$

имеет узлы (19), веса (21) и

$$
B=C=\frac{1}{2(n+1)},
$$

оценку остатка, равную значению (23).

Т е о ре м а 4. Единственная наилучшая на множестве $\mathbb{W}^{(2 r)} L_{2}$ квадратурная формула (27) имеет узлы (19), веса (21) и (28), оценку ошибки, равную значению (23).

Доказ ательство. Из теоремы 3 и [ [ $]$ следует, что единственной наилучшей на множестве $W^{(2 r)} L_{2}$ формулой вида

$$
\begin{aligned}
\int_{0}^{1} f(x) d x=B f(0) & +\sum_{i=1}^{r} \alpha_{i} f^{(2 i-1)}(0)+\sum_{k=1}^{n} A_{k} f\left(x_{k}\right)+C f(1)+ \\
& +\sum_{i=1}^{r} \beta_{i} f^{(2 i-1)}(1)+R_{n}(f)
\end{aligned}
$$

является формула Эйлера-Маклорена. Как известно (напр., [ $\left.{ }^{6}\right]$ ), веса этой формулы удовлетворяют соотношениям

$$
\alpha_{i}=-\beta_{i} \quad(i=1, \ldots, r) .
$$

Тем более формула Эйлера-Маклорена является единственной наилучшей на множестве $W^{(2 r)} L_{2}$ среди всех формул вида $\int_{0}^{1} f(x) d x=B f(0)+\sum_{k=1}^{n} A_{k} f\left(x_{k}\right)+C f(1)+\sum_{i=1}^{r} \gamma_{i}\left(f^{(2 i-1)}(0)-f^{(2 i-1)}(1)\right)+R_{n}(f)$. 
Отсюда и из $\left[{ }^{5}\right]$ следует утверждение теоремы.

3 а меч ание. Экстремальное свойство формулы Эйлера-Маклорена изучалось И. Шёнбергом [ $\left.{ }^{6}\right]$.

\section{ЛИ Т Е Р А Т У Р А}

1. Н и кольский С. М., Квадратурные формулы, М., 1974.

2. Н а й м а рк М. А., Линейные дифференциальные операторы, М., 1969.

3. Karlin S., Approximation with special emphasis on spline functions, New YorkLondon, 1969.

4. Karlin S., M icchelli C., Israel J. math., 405 (1972).

5. Ги рш о вич Ю., Изв. АН ЭССР, Физ. Матем., 25, 201 (1976).

6. S choenberg I. J., Theory and applications of spline functions, New York-London, 1969 , р. 157.

таллинский политехнический институт

\author{
Поступила в редакцию \\ 31/III 1975
}

\section{LEVIN, V. ARRO, J. GIRSOOVITS}

\section{MONEDE FUNKTSIOONIHULKADE PARIMAD KVADRATUURVALEMID}

On tuletatud parimad kvadratuurvalemid $(3),(1),(27) \underset{(2 r)}{\text { ja }}(27)$ vastavalt funktsioonihulkadele $W_{\boldsymbol{g}}^{(2 r)} L_{2}(2), W_{01}^{*(2 r)} L_{2}(24), \bar{W}_{91}^{(2 r)} L_{2}(25)$ ja $W_{01}^{(2 r)} L_{2} \quad(26)$.

\section{LEVIN, V. ARRO, Y. GIRSHOVICH}

\section{OPTIMAL QUADRATURE FORMULAE FOR SOME SETS OF FUNCTIONS}

For sets of functions $W_{g}^{(2 r)} L_{2}(2), W_{01}^{*(2 r)} L_{2}(24), \bar{W}_{01}^{(2 r)} L_{2}(25)$ and $W_{01}^{(2 r)} L_{2}$ (26) the optimal quadrature formulae $(3),(1),(27),(27)$, resp., are constructed. 